\title{
Foreword, with a Dedication to Vladimir Gerdt
}

\author{
Matthew England • François Boulier • \\ Timur Sadykov • Thomas Sturm
}

(C) The Author(s), under exclusive licence to Springer Nature Switzerland AG 2021

The International Workshop on Computer Algebra in Scientific Computing (CASC) is an annual meeting on computer algebra systems, the underlying algorithms for symbolic computation, and the role of both within scientific computing and its applications. CASC maintains a web presence at the following location.

\section{www.casc-conference.org}

In 2020, the 22nd CASC conference was planned to be held in Linz, Austria, at Johannes Kepler University (JKU). Due to the Covid-19 pandemic it had to be held as an online conference, hosted by the team at JKU. Although we were saddened not to be able to meet in Linz this year, we were grateful to the commitment of the organisers and participants that allowed CASC to go ahead successfully. This special issue contains selected work that was presented at this online event.

\section{Scope of CASC}

1. The theory of symbolic computation and its implementation in computer algebra systems. This includes, but is not limited to:

- new methods for exact computation;

- symbolic algorithms and their complexity;

\footnotetext{
M. England ( $\varangle$ )

Coventry University, Coventry, UK

e-mail: Matthew.England@ coventry.ac.uk

F. Boulier

Université de Lille, Lille, France

e-mail: Francois.Boulier@univ-lille.fr
}

T. Sadykov

Plekhanov Russian University, Moscow, Russia

e-mail: Sadykov.TM@rea.ru

\section{T. Sturm}

CNRS, Inria, and the University of Lorraine, Nancy, France

MPI Informatics and Saarland University, Saarbrücken, Germany

e-mail: thomas.sturm@1oria.fr; sturm@mpi-inf.mpg.de 
- automated reasoning in algebra and geometry;

- parallel symbolic computation;

- symbolic methods for differential, differential-algebraic and difference equations;

- symbolic methods for polynomial equations and inequalities;

- embedding of computer algebra within different software and hardware environments.

2. All other areas of scientific computing in regards to their benefit from or use of computer algebra methods and software. This includes, but is not limited to:

- the use of computer algebra in approximate computation;

- computer algebra methods in big data analytics;

- computer algebra methods for simulation and modelling.

3. Applications of the above throughout the natural sciences and engineering.

\section{Publication at CASC}

CASC has a long history of publishing conference proceedings as volumes of Springer's Lecture Notes in Computer Science (LNCS). The proceedings from CASC 2020 were recently published as volume 12291. Over the past two decades, CASC has evolved considerably, and both organizers and participants have desired additional forms of publication with respect to more extensive research that has reached some level of completion. Since CASC 2019 authors can choose in advance of the conference to either:

1. submit a paper for the LNCS proceedings; or

2. submit a shorter extended abstract which would be distributed only at the conference.

There are three audiences for the second option:

- Those who wish to talk on previously published work.

- Those whose work is not yet ready for publication, but wish to receive feedback on their work in progress.

- Those who plan to submit a journal paper on the work shortly.

This special issue was organised as a publication option for the third group. Submission was open a month after the meeting. From the thirteen authors with extended abstracts at CASC 2020, eight went on to submit articles for this issue. All papers then received at least three expert reviews and the editorial team choose to accept six of these papers, some after a revision stage to address reviewers' comments.

\section{Acknowledgements}

We wish to first thank the host institution of the CASC 2020 conference: Johannes Kepler University in Linz, Austria. They arranged a high quality online conference at short notice to benefit the CASC community in these difficult times. The local organisation team consisted of: Gabriela Danter, Manuel Kauers, Monika Peterseil, Veronika Pillwein, Clemens Raab, and Georg Regensburger. We also acknowledge the co-PC Chairs and Editors of the LNCS Proceedings, Matthew England and Evgenii V. Vorozhtsov; the co-General Chairs, François Boulier and Timur Sadykov; and the co-Publicity Chairs, Andreas Weber and Dominik Michels, and Hassan Errami who stepped into this role after Andreas Weber's untimely passing. Finally, we thank all of the reviewers of the papers in this issue for their time and knowledge which allowed the selection and improvement of the published papers: John Abbott, Alkiviadis Akritas, Changbo Chen, Frédéric Chyzak, Alicia Dickenstein, Beatriz Pascual Escudero, Vladimir Gerdt, Amir Hashemi, Hui Huang, David Jeffrey, Teo Mora, Gerhard Pfister, Gleb Pogudin, Daniel Robertz, Frank-Olaf Schreyer, Werner Seiler, Dingkang Wang, and Eva Zerz. 


\section{Dedication}

Sadly, our colleague Prof. Vladimir Gerdt passed away on the 5th January, 2021. He made numerous contributions to the fields of symbolic computation, differential algebra, and applications in physics. He has been deeply involved with CASC from the very beginning, and was serving on the series advisory board at the time of his passing. He was an excellent scientist and a kind-hearted and considerate man. We regret the loss to both science and his family.

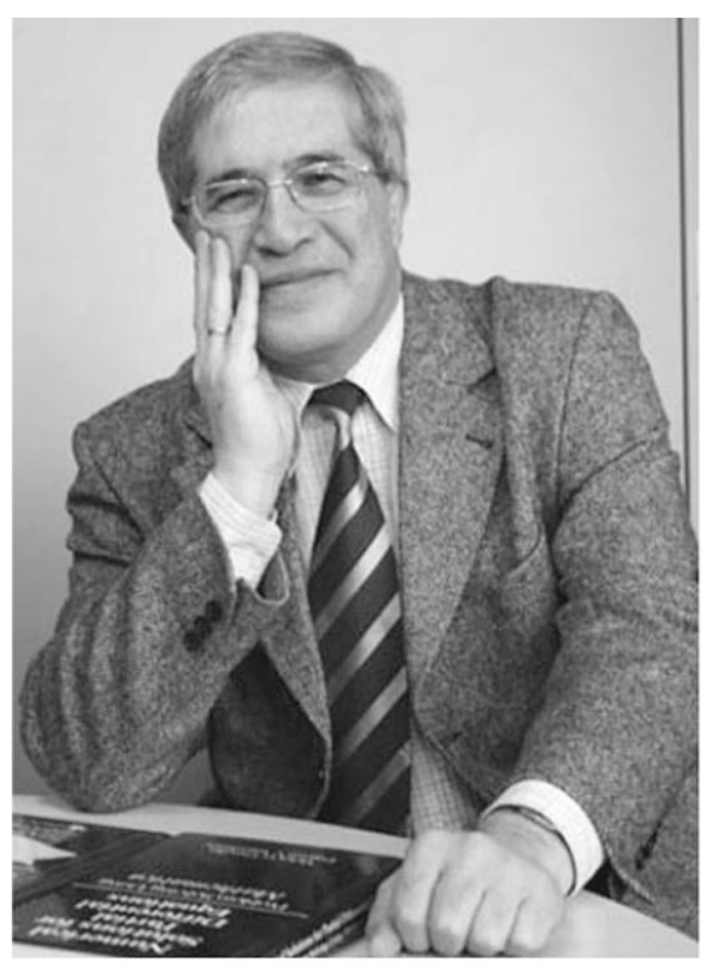

Prof. Vladimir Gerdt

Publisher's Note Springer Nature remains neutral with regard to jurisdictional claims in published maps and institutional affiliations. 